\section{Das neue Strahlenschutzgesetz - Überblick über die Auswirkungen auf das Fachgebiet der Radiologie}

\section{Einführung}

Strahlenschutz ist fast so alt wie die von Wilhelm Röntgen am 8. November 1895 entdeckten und nach ihm benannten Strahlen. Bereits kurze Zeit später erkannte man neben dem großen medizinischen Nutzen auch die schädigende Wirkung der Röntgenstrahlung. Bisher war der Strahlenschutz in Deutschland in den gefahrenabwehrrechtlichen Bestimmungen der Röntgenverordnung (RöV, für den Bereich der Röntgendiagnostik und Strahlentherapie unterhalb $1 \mathrm{MeV}$ ) sowie der Strahlenschutzverordnung (StrlSchV, für den Bereich der Strahlentherapie und Nuklearmedizin) geregelt. Am 27.6.2017 hat der Bundestag mit Zustimmung des Bundesrates das Gesetz zur Neuordnung des Rechts zum Schutz vor der schädlichen Wirkung ionisierender Strahlung beschlossen (BGBI.I S. 1966). In Artikel 1 dieses Gesetzes hat der Gesetzgeber das Gesetz zum Schutz vor der schädlichen Wirkung ionisierender Strahlung (Strahlenschutzgesetz - StrISchG) geregelt, welches der Umsetzung der Richtlinie 2013/59/Euratom des Europäischen Rates vom 5.12.2013 dient und überwiegend zum 1.10.2017 in Kraft treten wird. Das neu eingeführte Strahlenschutzgesetz zielt darauf ab, den Strahlenschutz zu verbessern, übersichtlicher zu gestalten sowie unnötige bürokratische Hemmnisse abzubauen.

Das neue Strahlenschutzgesetz wird sich auch auf das Fachgebiet der Radiologie auswirken. Zum einen fasst es als formelles Gesetz die Vorgaben zusammen, die bis dato in der auf dem Atomgesetz basierenden Strahlenschutzverordnung, der Röntgenverordnung sowie dem Strahlenschutzvorsorgegesetz geregelt waren. So werden zahlreiche Paragrafen identisch oder weitgehend deckungsgleich zu den bisherigen Normen in das Strahlenschutzgesetz inkorporiert. In formal-juristischer Hinsicht sind die Vorgaben daher nicht mehr nur als untergesetzliche Normen einzustufen, sondern sie werden durch das Reformgesetz normhierarchisch auf eine höhere Stufe verlagert. Dies hat Auswirkung auf die
Auslegung und Anwendung des Rechts. Kollidierten die Regelungen der Röntgenverordnung oder der Strahlenschutzverordnung mit höherrangigem formellem Recht, mussten sie nach früherer Rechtslage zurücktreten. Zukünftig stehen diese Regelungen auf der Ebene der Gleichordnung.

Zum anderem enthält das Gesetz zahlreiche Neuregelungen im medizinischen Bereich einschließlich der medizinischen Forschung, die im Folgenden vorgestellt werden sollen. Neben dem 1. Teil („Allgemeine Vorschriften“, §§ 1 bis 5) ist speziell der 2. Teil („Strahlenschutz bei geplanten Expositionssituationen“, $\S \S 6$ bis 91), welcher mehrheitlich die Belange medizinischer Strahlenexposition regelt, von Bedeutung.

Abgesehen von diesen Änderungen bestehen für das Fachgebiet der Radiologie die Regelungen der Röntgenverordnung sowie der Strahlenschutzverordnung - nunmehr im Gewand des Strahlenschutzgesetzes weitgehend fort.

\section{Zulässigkeit der Früherkennung asymptomatischer Patienten ( $\S 84$ StrlSchG, $\S 25$ Abs. 4a SGB V n. F., vgl. auch Art. 55 Abs. 2 lit. h RL 2013/59/Euratom)}

Die wichtigste Neuerung betrifft wohl die Zulässigkeit der Früherkennung von Erkrankungen asymptomatischer Personen. Derzeit ist die Mammografie-Untersuchung zur Früherkennung von Brustkrebs bei Frauen zwischen dem 50. und 69. Lebensjahr die einzige Reihenuntersuchung zur Früherkennung in Deutschland, bei der Röntgenstrahlung eingesetzt wird. Nach dem neuen Strahlenschutzgesetz sollen künftig auch individuelle Früherkennungsmaßnahmen, mithin außerhalb von Screening-Programmen zugelassen werden können. Den

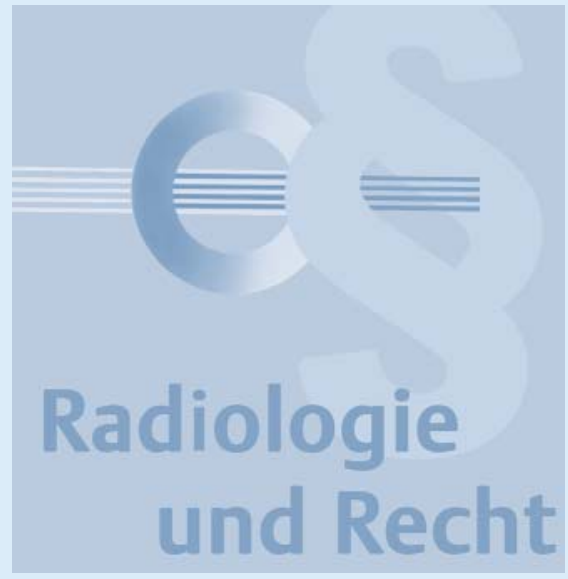

Begriff der Früherkennung wird §4 Abs. 16 StrISchG legal definiert als „Anwendung von Röntgenstrahlung oder radioaktiven Stoffen im Rahmen einer medizinischen Exposition zur Untersuchung von Personen, die keine Krankheitssymptome und keinen konkreten Krankheitsverdacht aufweisen (asymptomatische Personen), um eine bestimmte Krankheit festzustellen" Dazu könnten etwa Verfahren zur Früherkennung von Lungenkrebs, verengten Herzkranzgefäßen oder von Darmpolypen und Darmkrebs gehören. Zugleich werden jedoch hohe Anforderungen an die Rechtfertigung und die Umsetzung solcher Untersuchungen gestellt. Grundsätzlich steht der diagnostische und therapeutische Nutzen der betroffenen Person im Vordergrund. Voraussetzung ist daher, dass der Nutzen das Risiko der eingesetzten Strahlung überwiegt. Weiterhin muss die neuartige Früherkennungsuntersuchung

- ein wissenschaftlich anerkanntes Untersuchungsverfahren darstellen,

- zur Erkennung einer schweren Erkrankung im Frühstadium dienen und

- eine effektive Therapieform verfügbar sein.

Ob und wann derartige Untersuchungen zur individuellen Früherkennung zugelassen werden, hängt von der Entscheidung des Bundesministeriums für Umwelt, Bau, Naturschutz und Reaktorsicherheit ab das Gesetz enthält eine diesbezügliche Verordnungsermächtigung - und der Bewertung des Bundesamts für Strahlenschutz ab (vgl. § 84 Abs. 2 und 3 StrlSchG).

Sofern sich beide für eine bestimmte Früherkennungsuntersuchung aussprechen, 
hängt deren vertragsärztliche Erbringung zu Lasten der gesetzlichen Krankenversicherung allerdings davon ab, dass der Gemeinsame Bundesausschuss eine dahingehende Richtlinie bereits erlassen hat bzw. sie erlässt (§ 25 Abs. 4a SGB V n. F.). Ist eine Früherkennungsuntersuchung nach dem Strahlenschutzgesetz zulässig, können Kliniken und Radiologie-Einrichtungen eine zwingend auf fünf Jahre befristete Genehmigung bei der Strahlenschutzbehörde erwirken. Diese wird ihnen eingeräumt, wenn die nötigen (Qualitäts-) Voraussetzungen an Personal, Gerätetechnik etc. erfüllt sind (vgl. §13 Abs. 3 StrlSchG).

\section{Teleradiologie ( $§ 14$ Abs. 2 StrISchG)}

Ebenso hat der Bereich der Teleradiologie durch das neue Strahlenschutzgesetz umfangreiche Änderungen erfahren. Der Gesetzgeber hebt nunmehr den Aspekt der Regionalität hervor. Dies verdeutlichen die Vorgaben betreffend die „besondere[n] Voraussetzungen“ (§ 14 Abs. 2 StrlSchG). Demgemäß muss u. a. ein Gesamtkonzept für den teleradiologischen Betrieb vorliegen, das

- die erforderliche Verfügbarkeit des Teleradiologiesystems gewährleistet,

- eine im Einzelfall erforderliche persönliche Anwesenheit des Teleradiologen am Ort der technischen Durchführung innerhalb eines für eine Notfallversorgung erforderlichen Zeitraums ermöglicht; in begründeten Fällen kann auch ein anderer Arzt persönlich anwesend sein, der die erforderliche Fachkunde im Strahlenschutz besitzt,

- eine regelmäßige und enge Einbindung des Teleradiologen in den klinischen Betrieb des Strahlenschutzverantwortlichen stattfindet, welche die erforderliche Untersuchungsqualität auch bei komplexen und seltenen Untersuchungssituationen sicherstellt.

Intention des Gesetzes ist es mithin, das Regionalprinzip insgesamt zu stärken. Der Teleradiologe soll (nach Möglichkeit) in der Region des Untersuchungsortes tätig sein, damit er bei Bedarf persönlich verfügbar ist. Wo genau die örtliche Grenze zu ziehen ist, lässt sich derzeit nicht bestimmen. Für den Teleradiologen (oder seinen Vertreter) wird im Unterschied zur letzten RöV außerdem nicht mehr die volle Fachkunde mit 36 Monaten vorausgesetzt, sondern die erforderliche Fachkunde. Wie diese definiert wird, ist zur Zeit noch offen.

Ausweislich der Gesetzesbegründung (BTDrs. 18/11241, S. 255) soll auf diese Weise die Untersuchungsqualität gesichert und die Ausfallsicherheit gewährleistet werden, um eine kontinuierliche Patientenversorgung aufrechterhalten zu können. Daneben bezweckt das sog. Gesamtkonzept die Förderung der Transparenz gegenüber der Genehmigungsbehörde hinsichtlich der organisatorischen Strukturen (Aufgabenwahrnehmung, Zuständigkeiten und Weisungsbefugnisse). Zusätzlich zu den Anforderungen an technische Komponenten zum Informationsaustausch, die eine Datenübertragung nach dem Stand der Technik und den medizinischen Erfordernissen sicherstellen, sind daher auch organisatorische Festlegungen, die für den ordnungsgemäßen Betrieb und die Durchführung einer teleradiologischen Untersuchung relevant sind, offenzulegen (vgl. BTDrs. 18/11241, S. 256).

\section{Bewertungsverfahren Rechtfertigung ( $\S 6$ Abs. 1 StrlSchG)}

Neue Verfahren, bei denen Mensch und Umwelt ionisierender Strahlung ausgesetzt sein könnten - beispielsweise in der medizinischen Diagnostik -, müssen zukünftig das Bewertungsverfahren der Rechtfertigung durchlaufen. Dabei soll ihr wirtschaftlicher, gesellschaftlicher oder anderweitiger Nutzen abgewogen werden mit einer möglicherweise einhergehenden gesundheitlichen Beeinträchtigung. Diese Vorgabe ist eine besondere Ausformung des Rechtsfertigungsprinzips, welches das gesamte Strahlenschutzgesetz prägt. Die Einzelheiten dieses Bewertungsverfahren werden erst der noch zu erlassenden Verordnung zu entnehmen sein und lassen sich folglich gegenwärtig noch nicht abschätzen.

\section{Einführung des Medizinphysikexperten in der Röntgendiagnostik (§ 14 Abs. 1 Nr. 2b StrlSchG)}

An der Forderung einer entsprechend dem radiologischen Risiko abgestuften Einbindung und Verfügbarkeit eines Medizinphysikexperten hält das Gesetz fest. Das Strahlenschutz-gesetz sieht nunmehr - zusätzlich - verpflichtend vor, einen Medizinphysikexperten auch bei allen strahlendiagnostischen Untersuchungsverfahren und interventionsradiologischen Anwendungen, die mit hohen Dosen der untersuchten Person verbunden sind, hinzuzuziehen. Das Gesetz definiert den Begriff des Medizinphysikexperten in §4 Abs. 24 StrlSchG als „Person mit Masterabschluss in medizinischer Physik oder eine in medizinischer Physik gleichwertig ausgebildete Person mit Hochschulabschluss, die jeweils die erforderliche Fachkunde im Strahlenschutz besitzt“.

Demgemäß ist auch bei interventionsradiologischen und strahlendiagnostischen Verfahren mit einer erheblichen Exposition der behandelten bzw. untersuchten Person ein Medizinphysikexperte einzubinden. Die Neuregelung besagt jedoch nicht, dass er bei jeder Untersuchung zwingend anwesend sein muss. Ausweislich der Gesetzesbegründung (BT-Drs. 18/11241, S. 253) ist vielmehr eine regelmäßige Hinzuziehung des Medizinphysikexperten zur Optimierung und Qualitätssicherung der Anwendung und zur Beratung in Fragen des Strahlenschutzes erforderlich. Da insbesondere bei Röntgen-einrichtungen in der interventionellen Radiologie die genaue Art der durchzuführenden Anwendungen vielfach nicht bereits im Genehmigungs- oder Anzeigeverfahren bekannt sein wird, dürfte es geboten sein, die Einbeziehung des Medizinphysikexperten bereits zu fordern, wenn die Behandlung mit einer erheblichen Exposition verbunden sein kann. Daher darf die Einbindung des Medizinphysikexperten im Rahmen der interventionsradiologischen und strahlendiagnostischen Verfahren nicht mit derjenigen des Strahlenschutzes verwechselt werden. 
Eine noch zu erlassende Rechtsverordnung wird zu klären haben, „dass und in welchem Umfang ein Medizinphysik-Experte entsprechend dem radiologischen Risiko der Strahlenanwendung hinzuzuziehen ist sowie welche Untersuchungen mit radioaktiven Stoffen oder ionisierender Strahlung mit einer erheblichen Exposition der untersuchten Person verbunden sein können“ (§ 86 S. 2 Nr. 10 StrlSchG).

Da das Strahlenschutzgesetz der Umsetzung der Richtlinie 2013/59/Euratom dient, stellt diese eine wichtige Auslegungshilfe dar. Gem. Art. 83 Abs. 2 der Richtlinie sollen ihm u. a. folgende Aufgaben obliegen:

- Verantwortung für die Dosimetrie, einschließlich der physikalischen Messungen zur Bewertung der dem Patienten und anderen einer medizinischen Exposition ausgesetzten Personen verabreichten Dosis

- Optimierung des Strahlenschutzes

- Festlegung, Durchführung und Überwachung der Qualitätssicherung

- Kontrolle der Abnahmeprüfung

- Überwachung medizinisch-radiologischer Anlagen

- Schulung von medizinischen Fachkräften

- Beratung zur medizinisch-radiologischen Ausrüstung.

Aller Voraussicht nach wird sich diese Neuregelung speziell für den Teilbereich der Computertomografie und Strahlenanwendungen im Rahmen der interventionellen Radiologie auswirken

\section{Verlängerte Anzeigefrist (§19 Abs. 1i. V.m. § 20 Abs. 1 StrlSchG)}

Wer beabsichtigt eine Röntgeneinrichtung zu betreiben, die keiner Genehmigung bedarf, muss dies der zuständigen Behörde nicht - wie bislang - spätestens zwei Wochen ( $\$ 4$ Abs. 1 RöV a.F.), sondern nunmehr spätestens vier Wochen vor dem beabsichtigten Beginn schriftlich anzeigen.

\section{Medizinische Forschung (§§31 bis 37 StrlSchG)}

Das neue Gesetz hält weiterhin Neuregelungen für das Genehmigungsverfahren beim Bundesamt für Strahlenschutz für medizinische Forschung am Menschen vor. Im Schwerpunkt muss die Behörde entscheiden, ob der Einsatz von Strahlen medizinisch und ärztlich vertretbar ist. Jedoch sah die alte Rechtslage keine Fristen für die Entscheidung vor, infolgedessen sich das Genehmigungsverfahren bis zu über einem Jahr hinzog. Das Strahlenschutzgesetz sieht nunmehr konkrete Bearbeitungsfristen für den Genehmigungsantrag vor: Prüfung der Vollständigkeit des Antrags binnen 21 Tagen und Entscheidung binnen weiterer 90 Tage, es sei denn die zuständige Behörde hält die Verlängerung der Frist um maximal 90 Kalendertage wegen der Schwierigkeit der Prüfung für erforderlich. Zudem differenziert das Gesetz nunmehr zwischen dem Genehmigungsverfahren und dem bloßen Anzeigeverfahren. Gem. §31 Abs. 1 StrISchG unterliegt die medizinische Forschung unter Verwendung radioaktiver Stoffe grundsätzlich der Genehmigungspflicht, sofern sie nicht nur nach $\S 32$ StrISchG anzeigebedürftig ist. Entscheidend ist mithin, welche Bedeutung und Rolle der Strahlenanwendung im konkreten Forschungsvorhaben zuteil wird. §32 StrlSchG erfasst demzufolge den Fall der radiologischen Begleitdiagnostik. Auch hier hat der Gesetzgeber nunmehr eine straffe Fristenregelung in §33 StrlSchG normiert:

- Bestätigung des Eingangs der Anfrage innerhalb von 14 Tagen (ggf. Hinweispflicht wegen Unvollständigkeit) und

- Abschluss der inhaltlichen Überprüfung binnen weiterer 28 Kalendertage.

\section{Aufzeichnungspflicht (§ 85 Abs. 1 Nr. 3 StrlSchG)}

Das neue Strahlenschutzgesetz normiert im Vergleich zur bisherigen Rechtslage ferner umfangreichere Aufzeichnungspflichten des Strahlenschutzbeauftragten. Neben den Angaben zur rechtfertigenden Indikation, dem Zeitpunkt und der Art der Anwendung, dem erhobenen Befund einer Untersuchung sowie dem Bestrahlungsplan und dem Bestrahlungsprotokoll einer
Behandlung müssen die Aufzeichnungen zusätzlich Angaben zur Exposition

- der untersuchten oder behandelten Person oder zur Ermittlung dieser Exposition, einschließlich einer Begründung im Falle der Überschreitung diagnostischer Referenzwerte, sowie

- von Betreuungs- und Begleitpersonen, sofern ihre Körperdosis zu ermitteln ist,

enthalten.

\section{Informations- und Meldesystem für Vorkommnisse ( $\S 90$ StrlSchG)}

Auf Grund der zunehmenden Anwendung ionisierender Strahlung und radioaktiver Stoffe in der Medizin nimmt das Risiko von Fehlbestrahlungen und Unfällen zu. So kann es aufgrund technischer Mängel oder menschlichen Versagens beispielsweise vorkommen, dass ein Patient versehentlich mit einer zu hohen Dosis bestrahlt wurde und es in der Folge zu stärkeren Nebenwirkungen kommt. Derartige Vorfälle waren nach der bisherigen Rechtslage nur eingeschränkt meldepflichtig. Um die Patientensicherheit zu erhöhen und die Qualitätssicherung zu verbessern, normiert $\S 90$ StrlSchG nunmehr eine Meldepflicht von sog. bedeutenden Vorkommnissen (vgl. auch Artt. 63 lit. a, c und e, 96 RL 2013/ 59/Euratom).

Auf diese Weise sollen Meldungen über Fehlbestrahlungen und Unfälle gesammelt, ausgewertet und die daraus gewonnenen Erkenntnisse an andere Anwender weitergegeben werden. Ziel ist es, Gefahren bereits im Vorfeld zu erkennen und gegebenenfalls frühzeitig Gegenmaßnahmen einzuleiten. Das Problem an einem solchen Informations- und Meldesystem ist jedoch, dass der Arzt seinen Behandlungsfehler in gewissem Umfang selbst eingestehen muss. Ein derartiges „Selbstbezichtigungsrecht" ist aber nur schwer in Einklang mit der deutschen Rechtsordnung zu bringen, sodass begründete Zweifel an der Praktikabilität bestehen. Zwar begründet das Strahlenschutzgesetz selbst keine Haftung des Arztes, dem ein solcher Vorfall unterläuft. Jedoch ist nicht auszuschließen, dass der 
betroffene Patient auf diese Weise von dem Zwischenfall erfährt und auf diesem Weg beweisrechtliche Vorteile für eine zivilrechtliche Schadensersatzklage erlangt.

Einzelheiten zu dem Umfang der Meldepflicht, seinem Verfahren, zu ergreifende Maßnahmen, etc. sieht das Gesetz nicht vor. Stattdessen verweist die Norm auf die noch zu erlassende Rechtsverordnung. In der Rechtsverordnung kann insbesondere gem. § 90 StrlSchG festgelegt werden,

1. dass und welche Maßnahmen der Strahlenschutzverantwortliche einzuleiten hat, damit Expositionen bei einem solchen Vorkommnis so gering wie möglich gehalten werden,

2. dass und welche Maßnahmen der Strahlenschutzverantwortliche zu treffen hat, um solche Vorkommnisse zukünftig zu vermeiden,

3. dass und auf welche Weise der Strahlenschutzverantwortliche ein Vorkommnis aufzuzeichnen und zu untersuchen hat, dass und für welchen Zeitraum er diesbezügliche Aufzeichnungen aufzubewahren hat,

4. dass und auf welche Weise der Strahlenschutzverantwortliche der Aufsichtsbehörde

a) ein Vorkommnis zu melden hat,

b) Informationen und Erkenntnisse über Ursachen und Auswirkungen des Vorkommnisses sowie Maßnahmen zur Behebung oder Begrenzung der Auswirkungen des Vorkommnisses zu melden hat und

c) Maßnahmen zur Vermeidung von Vorkommnissen zu melden hat.

Außerdem hat der Strahlenschutzverantwortliche dafür Sorge zu tragen, dass bei einem derartigen Vorkommnis Name, Vornamen, Geburtsdatum und -ort, Geschlecht und Anschrift sowie Daten zur Exposition einer durch das Vorkommnis exponierten Person sowie zu den gesundheitlichen Folgen der Exposition unverzüglich aufgezeichnet werden. Diese Dokumentationspflichten dienen dem Zweck, die Umstände des Vorkommnisses rekonstruieren und die erforderlichen Schlüsse ziehen zu können. Sofern das Vorkommnis so gravierend ist, dass es zu gesundheitlichen Folgen für eine der exponierten Personen kommt, sind auch Angaben zu diesen Folgen aufzuzeichnen. Vorausgesetzt, dass es sich um ein meldepflichtiges Vorkommnis handelt und Maßnahmen zum Schutz der exponierten Person erforderlich sind, muss er die personenbezogenen Angaben zudem unverzüglich der zuständigen Behörde übermitteln.

\section{Strahlenschutzregister (§ 170 StrlSchG)}

Im Strahlenschutzregister werden Daten von Beschäftigten zusammengefasst und ausgewertet, die in ihrem Beruf erhöhter Strahlung ausgesetzt sind. Dazu gehören unter anderem auch Angehörige medizinischer Berufe. Das bestehende Register reicht bis in die 1990er Jahre zurück und umfasst derzeit Angaben zu etwa 400000 Personen, die strahlenschutzüberwacht werden. Um auch bei einem Namens- oder Arbeitsplatzwechsel von Personen, die mit ionisierender Strahlung umgehen, deren Schutz jederzeit lückenlos sicherstellen zu können, sieht das neue Strahlenschutzgesetz die Einführung persönlichen Kennnummer vor, die auch bei einem $\mathrm{Na}$ mens- sowie Arbeitgeberwechsel erhalten bleiben.

\section{Abschließendes Resümee}

Die Neuregelungen dienen der Verbesserung des Schutzes vor der schädlichen Wirkung ionisierender Strahlung und demzufolge der Patientensicherheit sowie der Qualitätssicherung. Vom Strahlenschutz werden in verstärkten Maße zukünftig aber auch Personen erfasst, die lediglich mittelbar in Kontakt ionisierender Strahlung gelangen, mithin das ärztliche wie auch das nicht-ärztliche Personal. Dieses Verständnis wird daher von entscheidender Bedeutung für die Auslegung und Anwendung des Gesetzes sein.

Die genauen Auswirkungen der Neuregelungen lassen sich derzeit noch nicht absehen, da der Gesetzgeber die konkrete Ausgestaltung der einzelnen Normen auf die noch zu erlassenden Rechtsverordnungen ausgelagert hat. Diese werden voraussichtlich erst im Laufe des kommenden Jahres in Kraft treten. Das Strahlenschutzgesetz selbst tritt hinsichtlich der Regelungen betreffend
- die Reihenuntersuchung zur Früherkennung ( $§ 84$ Abs. 2 und 3 StrlSchG),

- den Erlass der Rechtsverordnung zum Bewertungsverfahren der Rechtfertigung (§ 6 Abs. 3 StrlSchG),

- das Informations- und Meldesystem für Vorkommnisse (§90 Abs. 1 StrlSchG) sowie

- den Erlass der Rechtsverordnung zum Strahlenschutzregister ( $§ 170$ Abs. 10 StrlSchG)

am 1.10.2017 und im Übrigen, d. h. betreffend

- der Teleradiologie gem. §14 Abs. 2 StrISchG,

- des Bewertungsverfahrens der Rechtfertigung $\S 6$ Abs. 1 und 2 StrlSchG,

- der Einführung des Medizinphysikexperten in der Röntgendiagnostik gem. $\S 14$ Abs. 1 Nr. 2 StrlSchG,

- der verlängerten Anzeigefrist gem. § 19 Abs. 1i. V. m. § 20 Abs. 1 StrlSchG,

- der medizinischen Forschung gem. $\S \S 31 \mathrm{ff}$. StrlSchG,

- der Aufzeichnungspflicht gem. §85 Abs. 1 Nr. 3 StrlSchG und

- des Strahlenschutzregisters gem. $\S 170$ Abs. 1 bis 9 StrlSchG,

am 31.12.2018 in Kraft.

Prof. Dr. Peter Wigge

Rechtsanwalt

Fachanwalt für Medizinrecht

Dipl. jur. Jan Harald Schütz, LL.M.,

Wiss. Mitarbeiter

Rechtsanwälte Wigge

Scharnhorststraße 40

48151 Münster

Telefon: (0251) 53595 - 0

Telefax: (0251) 53595 - 99

E-Mail: kanzlei@ra-wigge.de

http://www.ra-wigge.de 